\title{
Credibility crisis in pediatric psychiatry
}

Our understanding of the neurobiology and treatment of psychiatric illness in children remains poor. Prominent
psychiatrists have now been accused of concealing the extent of their financial ties to the drug industry. We urgently
need to encourage more science in this area and we need vigorous regulation to restore some neutrality to the field.

A recent US Senate investigation has highlighted an ethics scandal involving prominent academic psychiatrists who study the efficacy of psychiatric medication, but who failed to disclose millions of dollars that they received from the pharmaceutical companies that make these drugs. Among the accused is Dr. Joseph Biederman, a renowned child psychiatrist at Harvard, who did not adequately declare over a million dollars of income that he received from pharmaceutical companies as consulting fees, calling into question the credibility and impartiality that he brought to several of the trials he guided. This ethical crisis is particularly dangerous in child psychiatry, as the potential consequences of treating the developing mind with powerful drugs are both less well understood and potentially more severe than in adults. The Senate's investigations should be a call to action by the federal government to step in and bring some objectivity to the business of medicating developing minds for psychiatric conditions.

Diagnosing mental disorders can be tricky even under the best of circumstances. Good biomarkers for psychiatric disorders (pediatric or adult) are nonexistent. Our knowledge of the neurobiology of these complex disorders also has glaring holes; the field is struggling to unravel the complexity of the genetic underpinnings of psychiatric diseases and the developmental factors that contribute to disease onset and progression. This is complicated further by frequent co-occurrence of several mental disorders and the paucity of good animal models to aid our understanding of the biology underlying these conditions. Psychiatrists must therefore resort to phenomenological diagnostic criteria. However, patients do not often map strictly to the consensus criteria set out for any one condition. Symptoms can also wax and wane. Diagnosis can be particularly problematic in children, where the diagnostic criteria are murkier and where the symptoms being presented can vary considerably from those observed in adults.

The treatment of many of these conditions is also plagued with problems. Although drug companies must demonstrate the safety and efficacy of their products before gaining approval from the US Food and Drug Administration (FDA), these trials are almost always done in adults. Adult findings do not necessarily translate well to children-we do not know the long-term effects of altering neurotransmitter levels on brain development and function. Once the drugs are approved and on the market, however, clinicians can prescribe them 'off label', or outside of their approved use, to children. Few antipsychotics are FDA approved for children, yet doctors increasingly turn to them or prescribe more than one in the hope that it may work.

Clinical trials are always difficult, and few companies want to take the added risk of testing children or putting them in placebo groups. In 1997, the US government tried to dangle a carrot to drug companies via the FDA Modernization Act, promising them an extra six months of marketing exclusivity if they were to conduct clinical trials in children. As a result of some of these trials, the FDA approved a 'black-box' warning for antidepressants in 2004, which included warnings about increased risks of suicidal thinking and behavior in children and young adults. Other trials were underpowered and inconclusive. The incentive for drug companies to invest in further long-ranging and well-controlled expensive pediatric trials seems meager. As it stands, many drugs are widely being prescribed off-label for children anyway; companies stand to lose income if the pediatric trials do not show the desired result and they have little to gain if they were to show an effect.

The revelation of the extent of the undisclosed ties between psychiatrists and drug companies further erodes the credibility of this field. Stanford University recently removed its chairman of psychiatry, Alan Schatzberg, as a principal investigator from a US National Institute of Mental Health grant investigating the use of a drug for depression, after the Senate investigation targeted him as having undisclosed ties to the company that was developing the drug. Industry funding of psychiatric research is not going to stop, and no one would advocate that there should be less money to fund research. However, universities and granting agencies should make much more of an effort to enforce their rules that require their employees or grantees to disclose their potential conflicts of interest. The US Senate is considering a bill that would require drug companies to report any payments to doctors that would exceed $\$ 500$ a year; steps such as these to increase transparency are also welcome.

More critically, however, there is an urgent need to put more science behind child psychiatry. We need an independent, objective assessment of the efficacy and safety of these medications, comparing existing generics and new products, and comparing non-drug or combination interventions to drug-only approaches. One option is to pool money from both industry and the government or other funding bodies, bringing together public and private money to fund such studies (similar to the 'Innovative Medicines Initiative' proposed by the European Commission). The raw data generated by clinical trials should be available for independent scrutiny. We also need to consider ways to increase recruitment in clinical trials, such as an alternate trial design where all patients initially get access to active treatment (Klein, D.F. JAMA 299, 1063-1065 (2008)) . Urgent action is needed to restore some objectivity and neutrality to this field; the stakes are simply too high to remain complacent. 